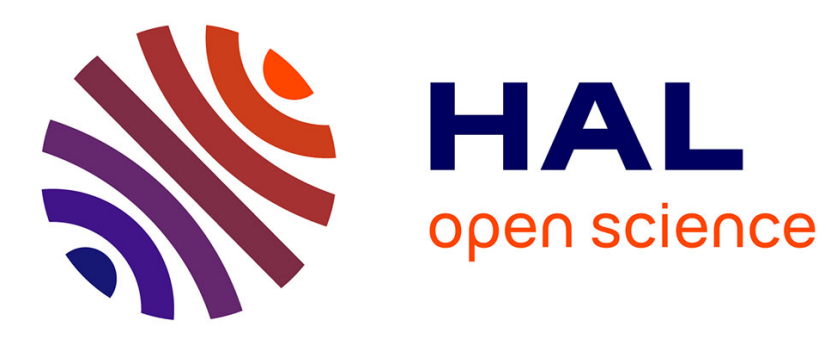

\title{
Diversité, conflictualités et sociabilités au cœur de la patrimonialisation de l'islam au Mali
}

\author{
Soufian Al Karjousli, Diama Cissouma, Anne Ouallet
}

\section{To cite this version:}

Soufian Al Karjousli, Diama Cissouma, Anne Ouallet. Diversité, conflictualités et sociabilités au cour de la patrimonialisation de l'islam au Mali. Joseph Brunet-Jailly, Jacques Charmes et Doulaye Konaté. Le Mali contemporain, Editions de l'Institut de Recherche pour le Développement, 2015, 9782709918312. halshs-01461671

\section{HAL Id: halshs-01461671 \\ https://shs.hal.science/halshs-01461671}

Submitted on 17 Feb 2017

HAL is a multi-disciplinary open access archive for the deposit and dissemination of scientific research documents, whether they are published or not. The documents may come from teaching and research institutions in France or abroad, or from public or private research centers.
L'archive ouverte pluridisciplinaire HAL, est destinée au dépôt et à la diffusion de documents scientifiques de niveau recherche, publiés ou non, émanant des établissements d'enseignement et de recherche français ou étrangers, des laboratoires publics ou privés. 


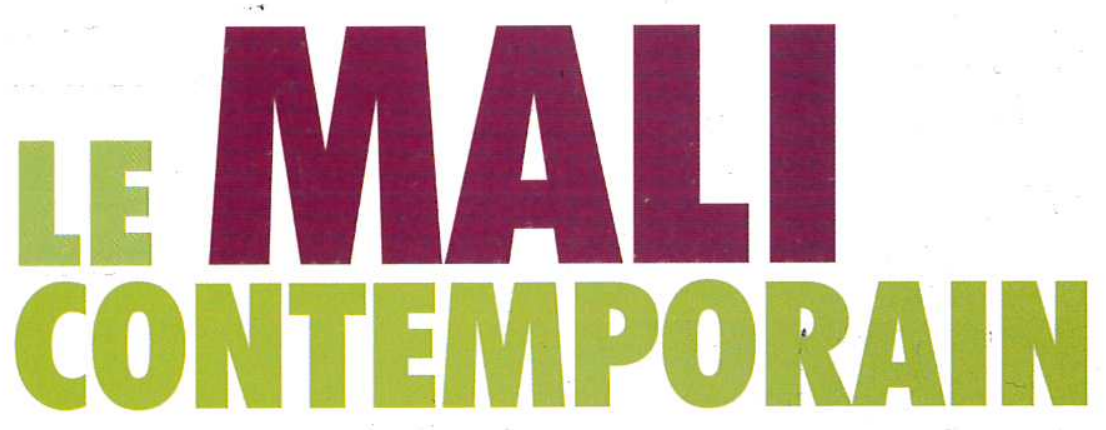

\section{Sous la direction de Joseph BRUNET-JAILLY Jacques CHARMES Doulaye KONATÉ}

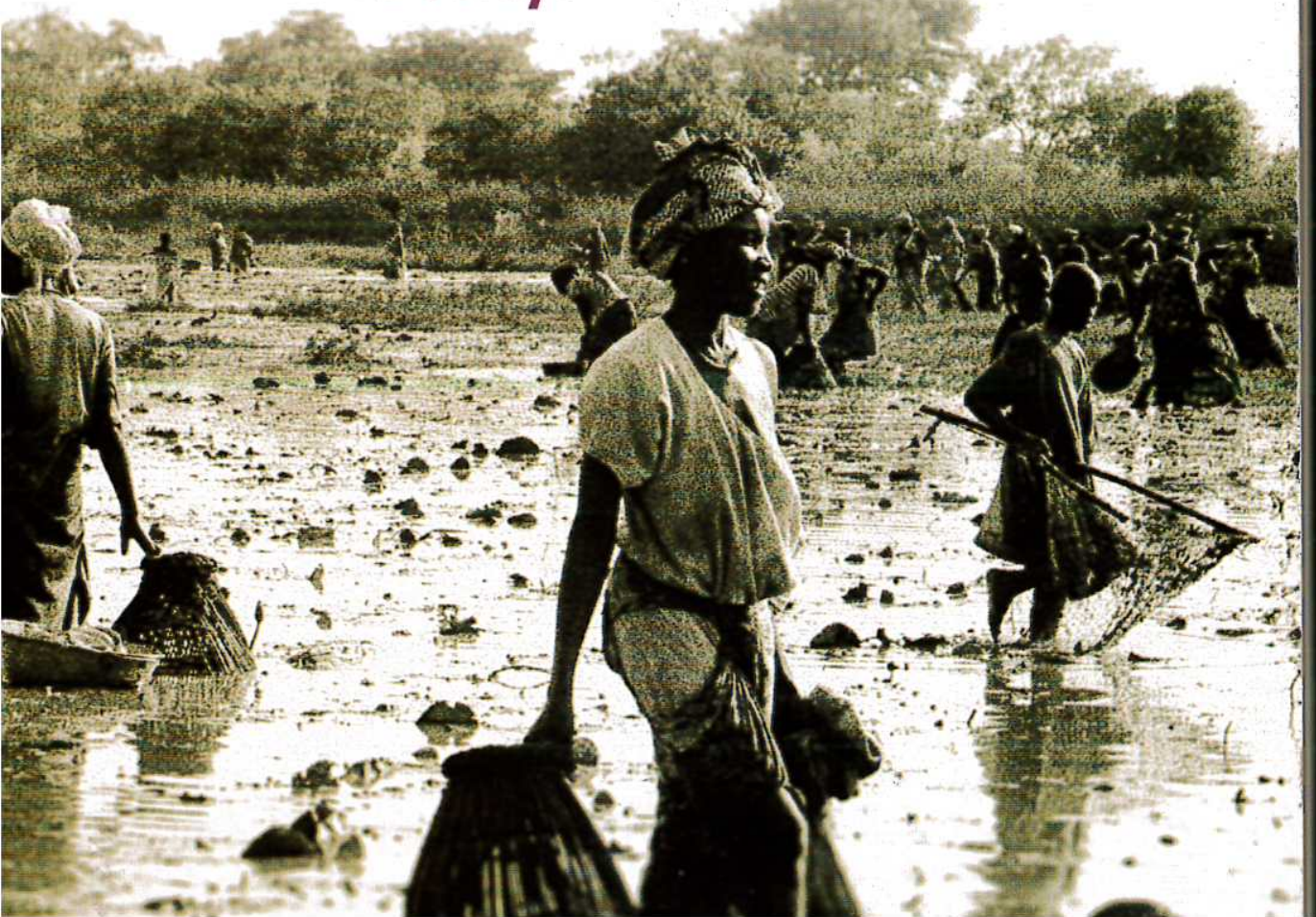




\section{Diversiné, conflictualinés ent sociubilinéés au cour de la patrimonialisation de Irislem เตอ Mali}

\section{Soufian AL KARJOUSLI}

\section{Diama CISSOUMA TOGOLA}

\section{Anne OUALLET}

On assiste actuellement à un redéploiement spectaculaire de la sphère religieuse, notamment autour d'un islam brandi à la fois comme un étendard de la refondation identitaire et comme soubassement du contrôle territorial. Or l'islam contemporain du Mali est marqué d'empreintes diverses qui renvoient à la fois à I'histoire, à des constructions mythiques et à des références importées issues des manœuvres géostratégiques de l'islam politique. Historiquement, au Mali, l'islam s'est construit à partir de différentes composantes, parfois en compétition, et il a accompagné de grands projets d'organisation socio-spatiale, tels que ceux qui ont permis l'établissement des empires du Ghana, du Mali ou encore du Songhoy. II s'y est généralement enrichi de connaissances, savoir-faire et pratiques locales. II a aussi parfois été utilisé comme fondement de projets politiques, comme celui de la Diina de Cheikou Amadou, et a, alors, servi de prétexte à des "rectifications ». Les références à l'islam sont donc incontestablement à resituer par rapport aux multiples héritages dans lequel il a évolué et qui l'amènent à être constamment reformulé.

Ces différents héritages, notamment ceux issus des encadrements politiques et sociaux d'une histoire longue se heurtent actuellement à des entreprises diverses promues par des acteurs qui tentent de s'approprier l'islam en en reformulant la mémoire. Des processus complexes de recomposition de l'espace et de la société ébranlent un bien commun construit au cours des siècles et conçu comme un véritable vivre ensemble. Des tensions éclatent au grand jour et accompagnent le phénomène de réislamisation de la nation. L'islam, à la fois mobilisé comme ressource patrimoniale et comme tremplin porteur d'innovations, s'enrichit des pratiques et savoirs locaux et participe pleinement à la redéfinition de l'espace commun de 


\section{Multiplicité des empreintes de Vislam}

L'islam contemporain du Mali se présente comme une mosaïque composée de plusieurs empreintes historiques, enrichie de créations locales, parfois doublées par des innovations importées. Cette mosaïque a des bases historiques, elle reflète en partie la diversité des doctrines qui se sont épanouies dans l'islam au fil des siècles, mais elle est également fortement marquée de fondements mythiques et légendaires.

La question des modalités de l'introduction de l'islam au Mali apparaît commeessentielle. Certains manuscrits anciens, les discours defigures emblématiques de l'islam et d'érudits permettent d'y apporter des réponses. Ils constituent les témoins historiques incontestables de la construction plurielle de l'islam, même si une grande partie de ces témoignages reste encore cachée entre les feuillets des manuscrits anciens, attendant des projets de sauvegarde, des analyses et des traductions, puisque la plupart d'entre eux sont en arabe ou en langues locales retranscrites en caractères arabes. Ces manuscrits sont les garants de la diversité et de la tolérance. Leur lecture permet de distinguer plusieurs versions de l'islam, renvoyant à des périodes, des interprétations et des cheminements différents, référant à des écoles de pensée, des érudits, des personnages charismatiques, mais aussi à des pratiques populaires qui inventent des formes personnalisées pour s'approprier l'islam selon les contextes locaux.

Les représentants traditionnels de l'islam et les nouveaux prêcheurs ont tous construit un discours sur la naissance de l'islam malien. Nos entretiens ${ }^{148}$ avec ces voix de l'islam dans différentes villes du Mali apportent des éléments de compréhension indispensables au décryptage des constructions intellectuelles reflétant les différentes composantes politico-religieuses.

Trois types de discours peuvent être identifiés selon la provenance qu'ils attribuent à l'islam malien. L'un insiste sur ses sources puisées à l'intérieur de l'Afrique. L'autre relie l'islam malien à une péninsule

${ }^{148}$ Ces entretiens ont été réalisés par les auteurs auprès des principaux représentants de l'islam dans les villes de Tombouctou, Bandiagara, Djenné, Ségou et Nioro lors de missions réalisées tout au long du programme de recherche "Mali contemporain " (soit en décembre 2009, janvier 2010, décembre 2010, janvier 2011 février-mars 2011 novembre 2011 Cortainc entrotionc ont ónalamont 
arabique élargie correspondant à l'actuel Proche-Orient, et se trouve des origines du côté de la Syrie et de l'Irak. Le troisième plaide pour une conversion de l'islam contemporain du Mali à l'islam mondialisé, qui est un islam déterritorialisé, donc au-dessus des frontières et à portée universelle. Ce dernier se prétend seul fidèle aux origines et s'est investi dù rôle de purger l'islam de ce qu'il considère être des contaminations intermédiaires liées aux influences locales, aux aires culturelles, et donc, par exemple pour le Mali, à toute trace d'africanité.

A propos des modalités d'introduction de l'islam en Afrique subsaharienne, l'une des pistes essentielles insiste sur le rôle prééminent de "Uqba ben Nâfi "Fahri dans la transmission de l'islam au sud du Sahara dès la fin du VII siècle. 'Uqba ben Nâfi' aurait été envoyé par Muawiyya ler, calife omeyyade de Damas, pour propager I'islam en Afrique. Les Kunta des régions de Gao et Tombouctou se présentent jusqu'à maintenant comme les descendants directs de ce général. Cette première période de diffusion de I'islam au sud du Sahara est tantôt décrite comme un temps de paix et de tranquillité sociale, tantôt au contraire comme une des époques les plus sanglantes pour l'islam en Afrique. L'islam s'est, en tout cas, dès cette époque, fondu avec les coutumes locales. La piste de Damas est régulièrement citée dans les récits mythiques des premières familles qui prétendent avoir eu un rôle éminent dans l'introduction de I'islam. À Ségou par exemple, les Djiré, reconnus comme étant ceux qui ont apporté l'islam dans la ville, se revendiquent un ancêtre syrien, sans remonter cependant jusqu'à l'illustre figure de 'Uqba ben Nâfi' lui-même.

Une autre piste insiste plutôt sur l'importance des Ibâdites venus d'Algérie au VIII siècle, grâce au concours de Cheikh Ali ben Yakhluf an Naftî (Ziyadé, 1991). Les populations converties auraient alors d'abord pratiqué le rite ibâdite avant d'adopter le rite malékite après la défaite de l'État Rustamide, qui occupait une partie de l'actuelle Algérie, contre les Idrissides (Hentati, 2004).

L'adaptation de cet islam de la première période à l'Afrique est visible jusqu'à maintenant à travers le Coran de Warch, dit de lecture occidentale ${ }^{149}$, qui reste un bel exemple d'intégration de la mélodie et 
d'imprégnation d'accents locaux dans les récitations coraniques. La naissance, puis la transmission de cette lecture coranique africaine, prouve l'insertion harmonieuse de l'islam à son arrivée en Afrique, et le Coran de Warch demeure à la base de la construction identitaire de nombre d'Africains jusqu'à nos jours. Cette filière maghrébine et transsaharienne aurait donc été portée par un islam qui, venu de la péninsule arabique, accompagnant les conquêtes, se serait fortement imprégné de valeurs locales.

Les discours qui valorisent un islam ancré dans le continent africain insistent, eux, sur la première Hégire réalisée par les compagnons du prophète de l'islam en Abyssinie. En effet, les premiers musulmans de la Mecque se sont réfugiés chez les chrétiens d'Abyssinie et ont été protégés par le roi chrétien d'Aksum (Al Karjousli 2010). L'Abyssinie, al Habasha, est alors désignée comme "le pays des vrais croyants". Un certain nombre de manuscrits et de discours présents au Mali reprennent cette épopée. Leurs auteurs et scribes valorisent - ou participent à diffuser- une conception très ouverte du musulmàn et de la croyance, s'appuyant sur des interprétations savantes du texte coranique. Ils reprennent notamment, souvent en les recopiant ou en les commentant, des ouvrages anciens dont certains du lettré As Suyûtî, par exemple ses œuvres intitulées respectivement Honneur aux Abyssins ${ }^{150}$ et La source des assoiffés de I'histoire des Abyssins ${ }^{15}$. On retrouve aussi L'histoire des grands personnages et l'histoire de la prophétie d'Ibn Sa'd ${ }^{152}$, Lasupériorité des noirs sur les blancs de Jahez ${ }^{153}$,

${ }^{150}$ As Suyûtî [né en 1445 aú Caire, mort en 1505], Rafi' chaan al habchân ("Honneur aux Abyssins "). Le manuscrit d'origine n'est pas daté, d'oủ l'habitude d'indiquer, quand elles existent, les dates de naissance et de mort de l'auteur pour le situer chronologiquement. La version utilisée est celle écrite par Zayn ed Dyn af Maghribi. Elle est datée de 1004 de l'hégire, soit 1596 du calendrier grégorien. Elle a été republiée deux fois récemment: par Muhamad Abdul Wahab Fadl, 1991, Université du Caire ; et par Muhammad Abdu Yamani, Safwan Dawudi et Hasan Abaji, 1994, Jeddah, Arabie Saoudite, Dar al Qibla li I thaqafa al islamiyya, 229 p. (version utilisée)

${ }^{151}$ Al Walwî Sadiq Sayyid b. Muhammad Manhal, 2001, Al 'Atchân fi Târikh al habchân («La source des assoiffés de l'histoire des Abyssins»), Éthiopie, 251 p.

${ }^{152} \mathrm{Ibn}$ Sa'd Abi Abdu-I-llah Muhammad ben Mani [né en 844], As-Syra an-Nabawiyya min al Tabaqât al Kubra ("La biographie du Prophète à travers les grands hagiographes "). La version utilisée ici a été publiée en 1989, au Caire, dar al Zahra li l i'lân al 'arabi, 2 vol., 907 p., à partir d'une version éditée à Leiden en 1904, ellemême réalisée à partir d'un manuscrit daté de 1199 . 
Éclaircir le rôle et l'image des Noirs et des Abyssins d'ibn Al Jawzi ${ }^{154}$. Des écrits récents, tels que ceux d'Al-Hâchem ${ }^{155}$ ou encore d'Al Sahhâr ${ }^{156}$ sont également très prisés.

Le discours véhiculé à travers ces ouvrages, souvent recopiés localement et/ou utilisés par des leaders charismatiques religieux, met en lumière le rôle de Bilal l'Abyssin, Bilâl al Habashi, muezzin du prophète. Ils posent généralement l'égalité entre blancs et noirs comme un des fondements de l'islam, mais certains sont tentés de proposer un renversement en s'appuyant sur les écrits de Jahez pour construire un discours de suprématie des noirs. Au-delà de la célébration du personnage de Bilal et de prophètes ou sages noirs tel que Job, les discours qui y font référence condamnent la dérive d'un islam qui se voudrait totalement arabe et blanc et dénoncent, par la même occasion, le retour d'un islam non égalitaire. C'est donc une théorie émancipatrice pour les non arabes, et recentrée sur des valeurs qui éliminent une vision raciale de l'arabité. La confrérie tijane, notamment à travers les Nyassènes, s'est en grande partie consolidée autour de cette dernière idée. Les écrits d'lbrahim Nyass ${ }^{157}$, qui ont, depuis le Sénégal, essaimé au Mali et jusqu'au Niger et au Nigéria à travers les réseaux nyassènes, transmettent cette vision.

Les manuscrits qui reviennent sur cette période, honorant la participation active de l'Afrique à la construction de I'islam en célébrant la première Hégire vers l'Abyssinie, et qui valorisent Bilal, sont également l'occasion d'alimenter largement le débat autour de vocables devenus sensibles dans leur définition, car ils sont jugés

rité des noirs sur les blancs "). En ligne, consulté le 24 juin 2014, http://www.alwaraq.net/Core/waraq/bibliography_indetail?id=102

${ }^{154}$ Ibn Al Jawzî [né en 1116, décédé en 1201], Tanwîr al ghabach fi fadl-i-s Sûdân wa al Habach ("Éclaircir le rôle et l'image des Noirs et des Abyssins"), republié en 1998, Al-Riyâd, Dar al-Sharîf.

${ }^{155}$ Al-Hâchem, B. Mohammad, 1998, Bilal, Muezzin du Prophète d'Allah, Paris, Al-Bouraq.

156'Abd al-Hamîd Jûdah al-Sahhâr, sans date, BilâlMu'adhin al Rasûl (« Bilâl, le Muezzin du Prophète "), Le Caire, dar Masr li I tiba'a, 200 p.

${ }^{157}$ Cheikh Hassane Ali Cissé, 1472 de l'Hégire, Saâdet al anâm bi aqwâl cheikh al islâm ("Le bonheur et les bienfaits aux gens par les paroles du Cheikh de l'islam »), éd. Kaolack, 119 p. Cheikh Hassane Ali Cissé imam de la Grande Moscuée do Módina 
fondateurs de la religion musulmane. Ces manuscrits véhiculent un discours qui expose une conception large et intégratrice d'un islâm compris comme soumission à Dieu, et d'un îmân comme croyance en Dieu. Par opposition, le kâfer, le mécréant, est alors défini comme " celui qui n'admet pas l'existence de Dieu ». Or ces compréhensions tendent actuellement à devenir minoritaires, particulièrement dans les discours dominants.

Pourtant les manuscrits anciens, certains d'entre eux en tout cas, ravivent la mémoire de l'islam pluriel proposant une option théologique intégratrice grâce à des concepts fondamentaux ouverts. Le musulman y est par exemple défini, non comme celui qui suit la seule religion musulmane, mais comme " celui qui se soumet à Dieu » reprenant la traduction étymologique du terme islâm ( $\mathrm{Al}$ Karjousli 2009b). Les vrais croyants, selon cette interprétation, ne se limitent pas seulement à ceux qui suivent la religion musulmane, mais incluent les chrétiens et les juifs rassemblés dans le texte coranique par l'expression "les gens du Livre », ahal al Kitâb. Ce sens réfère au fait que le prophète Muhammad avait envoyé ses adeptes chez les chrétiens de l'Abyssinie, appelés d'ailleurs par As Suyûtî « vrais croyants ». À partir de l'Abyssinie, c'est la branche de la Qâdiriyya qui aurait ensuite favorisé l'introduction de l'islam dans le reste de I'Afrique. Cela suggère une autonomie de la diffusion de l'islam à partir de la Corne de l'Afrique. Ces discours, repris notamment par certains qâdirî, transmettent l'idée qu'en Afrique, et donc en particulier au Mali, l'islam n'est pas parachuté, mais qu'intrinsèquement, le continent africain a été un vrai foyer où l'islam a germé et a grandi.

Les écoles de pensée et les rites véhiculés par l'intermédiaire des confréries et réseaux de l'islam sont très efficaces dans la transmission de la mémoire. C'est le cas de cette Qâdiriyya qui tire ses références d'Abdel Kader al Jilani (1077-1166), soufi originaire de Jilan (actuel Iran), mais ayant grandi à Bagdad. Elle est la confrérie la plus ancienne, s'est très fortement implantée dans la Corne de l'Afrique et dans la zone saharo-sahélienne où elle reste très présente, notamment en Mauritanie, au Mali, au Niger, au Soudan et dans le Nord du Nigeria, s'adaptant à chaque fois au milieu local. La chaine de transmission, silsila, s'avère essentielle au sein de cette confrérie. Elle remonte à Abdelkader Al Jilani, le même qui est célébré, dans un esprit d'ouverture aux différents mouvements de l'islam et de la chrétienté, 
Éthiopie dans la province du Balé, lieu où la légende locale place son tombeau. La Qâdiriyya est à présent discrète au Mali. Ses adeptes sont peu démonstratifs, sauf lors de rassemblements commémoratifs tels que celui de Hamdallaye, cité fondée par Cheikou Amadou et dont il ne reste que les ruines à une vingtaine de kilomètres au Sud-Ouest de Mopti, mais où se trouvent les tombeaux des proches de Cheikou Amadou.

À Djenné, I'héritage mémoriel de la Qâdiriyya demeure très vif au sein de certaines familles, notamment celles dont des membres se présentent comme des descendants de Cheikou Amadou, ou de ceux qui l'ont accompagné dans son djihad, et ce, même si depuis lors, ils ont embrassé une autre voie. C'est une mémoire qui les lie solidairement, et la portée de ces liens devrait pouvoir être décryptée grâce aux manuscrits anciens que certaines familles ont sortis, mais que d'autres hésitent encore à exhiber. La Qâdiriyya a régressé un peu partout, pendant et depuis la période coloniale. La confrérie semble avoir mieux résisté dans les campagnes. Ainsi, les zones rurales environnant Djenné et Bandiagara abritent encore une certaine influence qâdirî. Les références à Ousmane dan Fodio, chef qâdirî de l'empire du Sokoto (nord de l'actuel Nigeria) sont aussi très présentes à Gao.

Les manuscrits sont un lien essentiel dans la transmission de l'islam. Ils sont une source précieuse sur la pluralité de la pensée de l'islam, tout comme surle droit musulman et la vie quotidienne. Ils retransmettent aussi les débats sémantiques autour des interprétations du texte coranique. L'exemple de l'évolution des sens du vocable îmân le montre parfaitement: ce mot prend à chaque époque et avec chaque école de la pensée musulmane une dimension différente. Les lettrés musulmans ont, de tous temps, essayé d'associer la connaissance à la croyance, comme condition essentielle de cet îmân. "La croyance, al îmân, c'est la connaissance al-ma'ârifa »158. Ash Shahrastânî ${ }^{159}$ formule l'îmân en écrivant que celui qui a connu le savoir, puis l'a nié

${ }^{158} \mathrm{Al}$ Bukhârî [né en 810, décédé en 870], Jawahir al-Bukhâri ("Les diamants d'Al Bukhârî »), republié à Marseille, Dâr al-Fikr, 1922, p. 609.

${ }^{159}$ Abû al-Fath Muhammad b. Abd al-Karîm al-Shahrastânî [1086-1153], Al milal wannihal ("Les différentes écoles et inventions») publié en 1842, Printed for the Society for the Publication of Oriental Texts, London, 1846; la version utilisée ici 
par la parole, ne devient jamais incroyant, puisque la connaissance et le savoir ne disparaissent pas et ne s'effacent pas par de simples déclarations. II pense que l'inscription dans la voie de l'îmân devient une seconde nature.

Seule une étude plus poussée des manuscrits saharo-sahéliens pourrait nous dire si ce concept de la connaissance liant la connaissance et le savoir à la foi, concept central dans la Tijâniyya, est à interpréter dans la lignée de ces penseurs ou s'il résulte d'une évolution de pensée parallèle et indépendante de la Tijâniyya, mais aboutissant elle aussi à placer la connaissance au cour de la quête de cette confrérie dont les enseignements se retrouvent dans tout le Mali, notamment en milieu urbain. En effet, les villes qui étaient dominées par la Qâdiriyya, telles que Tombouctou ou encore Djenné, sont passées progressivement depuis un siècle à la Tijâniyya.

Cette voie tijane a offert un branchement sur de nouveaux réseaux, beaucoup plus adaptés, issus de l'Afrique du Nord - du Maroc exactement -, et se sont remarquablement étendus d'abord lors du djihad d'El-Hadj Oumar Tall. Ce djihad armé a été lancé en 1854 depuis le Sénégal. Ces réseaux ont ensuite prospéré tout au long du $X X^{e}$ siècle, en s'appuyant alors sur un djihad pacifique dont le but était une lutte intérieure et individuelle contre les mauvaises pensées et pour le surpassement de soi. Les réseaux tijane sont actuellement très présents du Maroc jusqu'au Niger. La filière oumarienne est particulièrement bien représentée à Bandiagara et à Ségou, où le patriarche de la ville est un représentant de la famille Tall.

Les voies confrériques ont joué un rôle dans la diffusion de la pensée mystique et du soufisme en Afrique de l'Ouest, mais ont aussi pesé sur l'organisation politique. Ces voies, turuq, ont tenu une place essentielle à travers l'engagement de leurs marabouts au moment de la colonisation (Robinson et Triaud 1997) et elles continuent à peser localement. Elles ont des capacités à réactiver certains réseaux. Ceux de la Tijâniyya oumarienne se réfèrent plutôt à des espaces de compromis, de sérénité et d'enseignement. La Hamawiyya et la Senussiyya, repérées comme porteuses de contestation à l'époque coloniale, se placent plutôt dans un espace du djihad et du conflit. 


\section{Conflictualités autour d'un patrimoine récusé}

Un peu partout donc, les manuscrits sont là, et ils sont gênants pour les tenants d'un islam exclusivement arabe et pour une conception de l'islam politique mondialisé qui dénie toute mémoire locale et qui ne supporte aucun écart aux règles hyper-normées que lui-même a définies dans sa conception exclusive. Cet islam «macdonaldisé » (Al Karjousli, 2009a) réduit la portée pluraliste de la pensée arabomusulmane, car il diffuse une religion simplifiée, basée sur cinq piliers définis comme immuables et incontournables, et quelques vocables phares auxquels toute polysémie a été ôtée.

Cet islam s'est développé au Mali depuis les années 1950 (Amselle, 1985) et a privilégié une conception qui rendait le musulman visible et identifiable par ses pratiques se démarquant du local par des pratiques codifiées à l'international, d'abord depuis l'Arabie à travers le wahhabisme, puis par des mouvements salafistes encore plus radicaux ayant des appuis au Pakistan, en Afghanistan ou encore plus récemment au Qatar. C'est aussi un islam de visibilité par ses marquages spatiaux à travers la construction de mosquées à l'architecture stéréotypée, sans correspondance avec l'architecture locale et recherchant à s'imposer, notamment par la distinction de mosquées du vendredi. Ces mosquées sont des édifices qui servent surtout au repérage signalétique de cet islam qui accompagne la mondialisation et elles sont donc situées, soit à proximité de grands axes, soit en situation d'espaces de conquête.

Le cas de Ségou est intéressant. La distribution des mosquées sur le territoire ségovien nous montre l'efficacité de ce marquage qui se fait à partir de la prolifération des mosquées du vendredi. Treize d'entre elles ont été dénombrées en 2011, dont deux wahhabites. La première mosquée wahhabite est située à proximité directe de l'axe goudronné Bamako-Ségou-Sévaré qui est l'axe de communication majeur du Mali. C'est typiquement une mosquée de la mondialisation sur laquelle surfe l'islam politique. La deuxième est située à Pelengana en position de front pionnier en frange urbaine sud-est de la ville, donc en situation d'accompagnement de l'extension urbaine. Les autres mosquées de vendredi se veulent être des mosquées de proximité pour faciliter la prière du vendredi dans les quartiers. 
Mosquées à Ségou, 2011

squées de Vendredi

Wahhabite

Auttes

be monsute anterieure

redo de El Hag Oonn

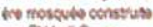

dar El Had Oamar

Autres mosqubes

Nans des casisiers
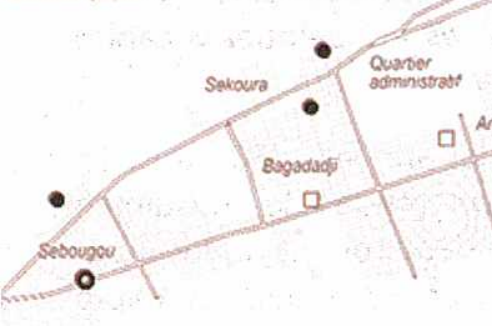
prosélyte : le terme da'wa signifie en arabe invitation, en l'occurrence invitation à devenir musulman, donc prosélytisme. C'est d'ailleurs la langue arabe que le mouvement adopte pour qualifier ses repères de base : le maqamya, de l'arabe maqam, le lieu, est le maillage de base de l'organisation et il devient effectif dès qu'il correspond à un comité islamique qui peut animer localement des réunions. Les femmes, qui n'ont pas le droit, elles, de dormir et de manger dans les mosquées, se regroupent autour de leur tahin, terme dont la traduction littérale de l'arabe est la farine.

Ainsi, la $D a$ 'wa investit et arabise progressivement les espaces de sociabilité du quotidien à partir desquels elle distille son message. Les Da'whî s'introduisent par exemple dans les grins, espaces de sociabilité de base au Mali, et y entreprennent bientôt des démarches individuelles pour convaincre les uns et les autres. Les $D a^{\prime}$ whî n'hésitent pas à critiquer l'autoritarisme et la manière brutale des Wahhabites, concurrents hanbalites directs dont ils analysent les comportements comme étant moins persuasifs et contraires au prosélytisme. Selon eux, c'est l'éloquence et l'art de convaincre qu'il convient de travailler. Inversement, les Wahhabites considèrent les pratiques de la Da'wa comme déviation, bid'a.

Au-delà de ces mouvements, c'est en fait une véritable réislamisation de la société (Holder 2009) qui s'opère. Elle se traduit globalement par la multiplication de nouvelles offres religieuses, la réactivation d'offres déjà existantes et la compétition entre toutes ces propositions. On observe aussi, indéniablement depuis quelques temps, une politisation de la sphère religieuse musulmane, ce qui vient encore démultiplier les offres. Les femmes, les jeunes et de manière plus globale les cadets sociaux, y cherchent leur place. II est connu que ce sont notamment les femmes qui ont supporté I'association Ançar Dine International de Chérif Ousmane Madani Haïdara, dont la popularité a gagné jusqu'à Djenné, pourtant réputée être le bastion de marabouts redoutés et respectés pour leurs pratiques ésotériques. L'objectif d'Ançar Dine International était de s'étendre dans le septentrion malien, mais son élan a été brisé par l'invasion djihadiste et la confiscation du devant de la scène par une organisation homonyme, Ansâr ed-Din, conduite par lyad Ag Ghaly, et portant un islam plus radical. 
De nombreux nouveaux soufis tentent aussi leur chance. Certains sont devenus de véritables entrepreneurs religieux, à l'image de Soufi Bilal, ou encore de Soufi Adama, qui opèrent à partir de la capitale, Bamako, et tentent de capter une clientèle extrêmement labile dans ses affiliations (Bourdarias 2009). D'autres soufis ont une audience plus limitée et locale. C'est le cas de Soufi Hamma et de Bassidy Tounkara qui œuvrent à Djenné.

Cette réislamisation de la société se manifeste non seulement par le marquage de l'espace, mais aussi par son influence sur des aspects très visibles de la vie quotidienne et sur le langage. Ainsi, l'apparition de nouvelles pratiques vestimentaires importées marquent les espaces du quotidien et provoquent des tensions à partir du moment où elles tendent à s'imposer comme le religieusement correct. De même les codes importés de la prière et de pratiques nouvelles sont récupérés comme une façon de marquer la différence et de se poser en défiance des autres ${ }^{160}$, qui sont alors dénoncés comme touchés par la déviance, bid'a.

Parallèlement, la nouvelle dynamique religieuse semble se resserrer sur quelques notions qui envahissent la sphère publique, jusqu'à la sphère langagière, où un certain nombre de repères deviennent des piliers normatifs. Ainsi des vocables de base islâm, îmân, kâfer ou encore jihâd, bay'a, takfir, bid'a, s'imposent progressivement comme incontournables, après avoir été vidés de la diversité des interprétations qu'une pensée islamique pluriséculaire leur a léguées, comme on l'a vu précédemment à propos du vocable islâm. Si tous ces concepts ont fait l'objet de discussions dans les manuscrits anciens, ils cristallisent à l'heure actuelle des tensions, des conflictualités très importantes, jusqu'à s'imposer parfois de manière très violente dans la conception d'un djihad guerrier et sanglant : celui, par exemple, qui a été pratiqué par le MUJAO ou encore par Ansâr ed-Din en 2012 et 2013.

Ces préceptes très encadrés de comportements assortis d'obligations sont formulés par l'islam « macdonaldisé », de manière plus ou moins

160 Doucouré Yacoub, 2010, Mulahazât 'ala arâ'ba'd el 'ulamâ (« Remarques sur les différents avis des savants »), dont un chapitre s'intitule Jawâz al Isdal wal Qabid fi-l Salât (" Une position vis-à-vis du regroupement ou du relâchement [des bras pendant les prières] »), publié à Sikasso, Mali, p. 25. L'imam Yacoub Doucouré, qui 
violente selon les contextes d'imposition. Ils ont en commun de piétiner toute autre forme.de pensée et toute référence à d'autres écoles de pensée qui ont pourtant été puissantes dans la civilisation musulmane et dont on retrouve, pour certaines en tout cas, les enseignements à travers les manuscrits ou les pratiques ancrées en Afrique de l'Ouest. Certains concepts fondateurs d'une civilisation musulmane qui s'est enrichie de ses contacts et de ses conquêtes disparaissent entièrement au profit d'une simplification permettant un meilleur contrôle du musulman qui est totalement confondu avec le croyant (Al Karjousli 2006). Dans la nouvelle version uniformisée, islâm et îmân sont alors fusionnés en imposant un transfert du contenu de l'îmân sur l'islâm grâce au procédé bien connu de la synonymie. L'utilisation outrancière de cette synonymie produit des amalgames. C'est là un grand danger qui a d'ailleurs été pointé depuis des siècles par un certain nombre de grammairiens arabes, tels que As Suyûtî ou encore Ibn Fâres ${ }^{161}$. Quelques manuscrits subsahariens plaident aussi pour le respect de la polysémie, c'est le cas de celui-ci, appartenant à I'Institut des Hautes Études Islamiques Ahmed Baba de Tombouctou :

Ma-t tahada lafzuhu wa-kh talafa maanahu « Ce qui est unifié par la prononciation et différencié par le sens »
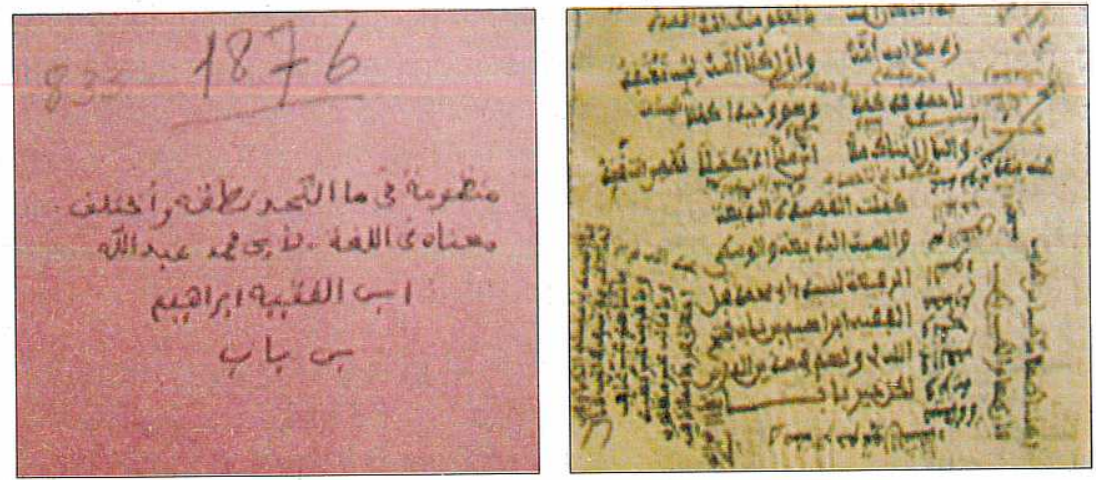

Auteur: Abi Muhammad Abdullah Ibn al Faqyh Ibrahim

IHEl Ahmed Baba, Tombouctou, Manuscrits $n^{\circ} 1876$ et $n^{\circ} 833$

${ }^{161}$ Ibn Fares, [mort en 1004], Kitâb as sâhibi fy figh al lugha al arabyia wa masâiliha wa sunan al arab, (" Le livre du compagnon sur le savoir linguistique de la langue arabe et de ses règles langagières »), retravaillé par Muhammad Ali Baydun, 1997, 


\section{LE MALI CONTEMPORAIN}

A côté de la synonymie, le resserrement monosémique est le deuxième procédé classique utilisé par l'islam réformateur du prêtà-penser mondialisé. II se cale alors sur un seul sens qui est présenté comme le seul vrai. La régression de la polysémie se double ainsi d'une fixation sémantique de certains vocables-clés auxquels on impose un sens unique, ce qui sert à véhiculer les préceptes diffusés par l'islam politique au pouvoir ici et là au Proche-Orient. C'est ainsi que, peu à peu, s'impose par exemple une conception du cafre (kâfer), à laquelle n'échapperait que le seul « vrai » musulman formaté selon les nouveaux principes édictés par l'islam conquérant de notre siècle. La conception montante du kâfer est pourtant en parfaite opposition avec l'acceptation de l'Autre et avec les pratiques de partage en usage au Mali, par exemple à travers les échanges socioéconomiques et festifs entre chrétiens et musulmans. Cette nouvelle définition va jusqu'à rejeter dans la catégorie de cafre les chrétiens, les juifs et même de nombreux musulmans dont les pratiques ne seraient pas jugées conformes. Chrétiens, juifs, musulmans et mệme zoroastriens sont pourtant considérés, dans les écoles majoritaires de la pensée musulmane, comme gens du Livre, ahl al-Kitâb.

Ceci dit, il faut reconnaître que ces acceptions restrictives de concepts et ce rejet totalitaire de ce qui n'est pas, à un moment donné, le religieusement correct, ne sont pas seulement des importations récentes. L'islam a toujours été traversé de courants plus ou moins ouverts à l'Autre et certaines propositions actuelles trouvent leur écho dans une histoire locale marquée de djihad entre musulmans, par exemple les affrontements entre El-Hadj Oumar Tall et Cheikou Ahmadou au XIXe siècle, ou encore les initiatives de Cheick Ousmane dan Fodio (1754-1817), fondateur du califat de Sokoto et initiateur d'un djihad contre le roi du Gobir en 1804. -

Il convient aussi de rappeler que la division règne également parmi les érudits, et que, par exemple, I'histoire de I'islam en Afrique a également été marquée par des penseurs tels que Maghili, lettré originaire de Tlemcen qui a fait office de conseiller d'Askia Mohamed à la fin du XVe siècle. Maghili semble avoir joué un rôle important dans I'islamisation du Songhoy, comme nous l'apprennent certains manuscritsprésentsàTombouctou, dontlescélèbres Questionnements d'Askia Muhammad et réponses d'Al Maghîlî (Hunwick, 1985). II a aussi prodigué des conseils au Roi de Kano (royaume Haoussa) en 1499 
ainsi qu'en atteste un manuscrit retrouvé à Ibadan $^{162}$ sur Le droit des dirigeants à empêcher les gens de faire ce qui n'est pas permis. La pensée radicale de Maghîlî était particulièrement attachée à plaider I'intolérance à l'égard des Juifs, allant jusqu'à la persécution ${ }^{163}$.

Si les sources d'un islam réformateur existent localement, elles sont actuellement surpassées par une vague ultra-radicale qui est importée et compte sur la désintégration de territoires faisant sens historiquement ou géographiquement (Di Méo 1998), donc construits par les encadrements historiques, appropriés par les populations, et relevant d'un patrimoine vécu (Cissouma et Ouallet, 2007). Ces territoires sont des espaces pleins de mémoires et construits autour de projets politiques qui ont laissé des marques à travers lesquelles les populations peuvent se reconnaître. Ce sont certains de ces éléments qui ont été repérés comme patrimoniaux par les politiques officielles actuelles, et ont fait l'objet de labellisations encouragées au niveau international par I'UNESCO. II en est ainsi par exemple des villes anciennes de Djenné, des trois célèbres mosquées et des tombeaux des saints de Tombouctou, ou encore du Tombeau des Askia de Gao qui ont été inscrits sur la liste du patrimoine de l'humanité.

De tels symboles sont inacceptables pour un islam mondialisé qui travaille à effacer ce type d'empreintes. La destruction des symboles montrant à la fois l'enracinement local des cultures et la reconnaissance internationale par le label du patrimoine mondial de I'UNESCO est l'expression la plus visible de cette volonté de gommer toute appartenance particulière. En fait, au-delà, c'est toute la formulation locale des cultures qui est refusée et combattue, car elle est source de résistance au projet de cet islam politique mondialisé, qui s'appuie sur l'effacement de la mémoire locale. Le label UNESCO est tout spécialement interprété par l'islam salafiste comme un marquage concurrent. Le dynamitage des bouddhas de Bamyan, dans le centre de l'Afghanistan, détruits en mars $2001^{164}$, comme ensuite la destruction systématique des tombeaux des saints à

${ }^{162}$ Manuscrit présent à l'université d'Ibadan, sous le $n^{\circ} 212 / 82$ et publié aux éditions Al Furqan, 2000, Londres.

${ }^{163}$ Ce qui lui vaut d'être parfois appelé le « Hitler algérien », comme le rapporte l'Observatoire de l'islamisation du 16 août 2011. En ligne, consulté le 24 juin 2014, http://www.islamisation.fr/apps/search/?s=maghîlî 
Tombouctou le 30 juin 2012 et les jours suivants, en sont l'expression concrète. Au Mali, le porte-parole d'Ansâr ed-Din a d'ailleuis très clairement expliqué que le passage à l'action rapide des combattants islamistes pour la destruction des mausolées de Tombouctou avait été motivé par l'inscription des biens classés de Tombouctou sur la liste du patrimoine mondial en péril, inscription à laquelle I'UNESCO avait procédé le 26 juin $2012^{165}$, soit quelques jours avant le saccage. Ces destructions sont des manifestations paroxystiques et tragiques nées d'un refus de reconnaître un patrimoine hérité localement et construit depuis les générations passées. Elles visent au contraire à imposer, par la force, des normes standardisées autour d'un islam politique s'appuyant sur des références importées.

Les relations entre le patrimoine et la religion se tissent plus habituellement sur un mode beaucoup plus apaisé (Cissouma et Ouallet 2009). En effet, jour après jour, les habitants sont amenés à formuler des propositions de vivre ensemble, à proposer des adaptations et innovations qui viennent enrichir l'islam et surtout à rester attentifs pour préserver le lien social.

\section{Diversité, innovations ef sociabilitiés au coeur d'un Islam vécu comme patrimoine au Mali}

Le vivre ensemble est un bien commun historiquement construit et consolidé à travers des réappropriations successives, qui intègrent des innovations. Ce vivre ensemble est au cœur de formes de sociabilités qui passent très souvent par des manifestations collectives, dont les célébrations religieuses, mais débouchent aussi sur des actions concrètes de solidarité, d'échanges, de travaux collectifs, par exemple de préservation du patrimoine lors des fêtes de crépissage des mosquées. Ainsi à Djenné la mosquée apparaît non seulement comme un repère important en tant qu'édifice unique, architecturalement exceptionnel, et qui participe à la célébrité de la ville, mais elle est aussi porteuse d'enjeux pour une communauté d'habitants qui se définit d'abord comme musulmane (Cissouma et Al Karjousli 2014). L'islam comme culture est perçu dans cette ville 
comme un socle fondateur sur lequel se bâtit la société de Djenné (Holder 2004) et qui permet à la mémoire collective de tester la cohésion sociale nécessaire à l'entretien de la mosquée pendant sa réfection annuelle. De nombreuses rivalités existent, mais la capacité à les faire taire pour cet événement annuel est, pour la ville entière, un test à renouveler, et il est renouvelé annuellement.

Les manifestations festives sont un moyen très efficace de mobilisation collective et de convivialité, emblématiques du vivre ensemble. Les villes saintes du Mali qui sont des espaces de référence de la culture malienne fortement liée à l'islam sont des espaces privilégiés pour ces manifestations parce qu'elles donnent un territoire à la mémoire et aux valeurs sur lesquelles s'appuie la volonté de continuer à vivre ensemble. Un certain nombre de ces manifestations se déroulent autour de bâtiments qui structurent fortement les espaces. II en est ainsi par exemple de la mosquée de Djenné, ou encore des trois plus anciennes mosquées de Tombouctou (Djingareyber, Sankore et Sidi Yahya) ; mais aussi à Gao du Tombeau des Askia, autour duquel précisément les jeunes se sont mobilisés, pour en assurer la protection pendant l'invasion djihad:ste.

Cependant, ces expressions collectives peuvent également prendre comme support des espaces non monumentaux, tout aussi porteurs de sens, qu'ils soient ressentis comme lieux d'espoir ou lieux de mémoire. C'est le cas à Nioro, Dilly, Hamdallaye autour des tombeaux, ou encore à Déguembéré près de Bandiagara, où est célébrée la disparition d'El-Hadj Oumar Tall dans la caverne. Cela fait l'objet de ziyara qui ont lieu soit au moment de fêtes musulmanes, soit se calent plutôt sur la célébration de saints pour les adeptes de la Qâdiriyya, les deux événements pouvant d'ailleurs coïncider. À Nioro, le marquage se fait autour de l'organisation des fêtes religieuses des hamallistes et de la Tijâniyya oumarienne. La cohésion sociale s'y articule autour d'une seule mosquée de vendredi. Les différents espaces sont marqués par des activités culturelles, des danses, des chants religieux, et des activités économiques dont la coexistence n'est nullement occasion de conflits. Djenné, Tombouctou, Ségou et Nioro sont des espaces de concentration de nombreuses rencontres, manifestations qui associent convivialités, démonstrations religieuses mais aussi échanges économiques, ceux liés par exemple à l'expression artisanale ou encore à la mise en valeur de la culture nour des offres tourictiaues 
L'appropriation des espaces se fait sous différentes formes : prières, pèlerinages, activités commerciales de façon individuelle ou à partir de regroupements de ressortissants, de sympathisants ou de fidèles, notamment pendant les jours de fête tel que le Maouloud ou encore la "Nuit du Destin », Layla-t-ul Qadr. Ainsi, le Maouloud de Tombouctou était réputé comme un moment de partage, de rassemblement et comme un espace de liberté exceptionnel et repéré comme tel dans tout le Mali. Beaucoup de Maliens ont ainsi plaisir à rappeler $q^{\prime}$ ' "à Tombouctou, pendant le Maouloud, tout est permis ». La prescription d'une charia fondamentaliste dans les espaces occupés a particulièrement pris pour cible ce genre d'événement qui allie le festif, la culture et l'islam, favorise les rencontres et les échanges. Un certain nombre de mouvements de l'islam réformateur s'en font actuellement les relais à travers tout le Mali.

Ces moments festifs de rassemblement figurent parmi les éléments repérés comme garants de la cohésion sociale. Au-delà, le système de transmission des savoirs et de la connaissance est également repéré comme nécessaire à préserver pour la pluralité de la refondation malienne. La transmission des connaissances passe par un ensemble de pratiques et d'enseignements qui ne sont pas intégrés dans le système de l'enseignement institutionnel, mais qui demeurent un patrimoine vivant, en tout cas dans un certain nombre de lieux emblématiques. Ainsi, à Djenné, comme à Tombouctou, les maitres d'écoles coraniques affirment que la perpétuation du système de l'éducation coranique, actuellement fortement ébranlé au Mali, est un enjeu majeur pour la préservation de la culture et un enjeu pour le développement de leur ville. À Djenné, les savoirs maraboutiques ésotériques sont aussi désignés par de nombreux habitants comme un patrimoine immatériel à préserver et à intégrer dans un programme global de développement permettant de transmettre l'image de la ville en tant que haut lieu maraboutique et ville de savoir.

Quant aux manuscrits anciens, ils ont été repérés de façon institutionnelle comme un patrimoine à part entière, même si leur préservation est délicate à mettre en œuvre. De nombreux acteurs nationaux et internationaux se mobilisent pour leur sauvegarde et des cris d'alarme ont été lancés lors de leur mise en péril par les islamistes. Ils sont une source de références inestimables, par exemple sur les savoir-faire en termes de régulations des conflits. Certains manuscrits renseianent sur la résolution de conflits sous 
les Askia ou encore à l'époque d'Ousmane Sourate de la Fâtiha dan Fodio, mais ils illustrent aussi parfois de Zabûr la capacité de l'islam local à innover. Un manuscrit de Tombouctou qui reprend la Fâtiha le montre. Celle-ci se nomme "Fâtiha de Zabûr » (Fâtihatu I Zabûr). Elle fait référence au Livre des psaumes, au Livre de David. En effet, cette Fâtiha, sourate d'ouverture du Coran, fonctionne dans ce manuscrit comme un pont entre la Thora et le Coran. Nous sommes ici au cœur de la création théologique de la pensée malienne qui met en valeur les liens possibles entre judaïsme ${ }^{166}$ et islam.

La lecture d'une autre Fatiha, trouvée à Djenné, nous montre la capacité d'utilisation de cette Fatiha pour recevoir la baraka.

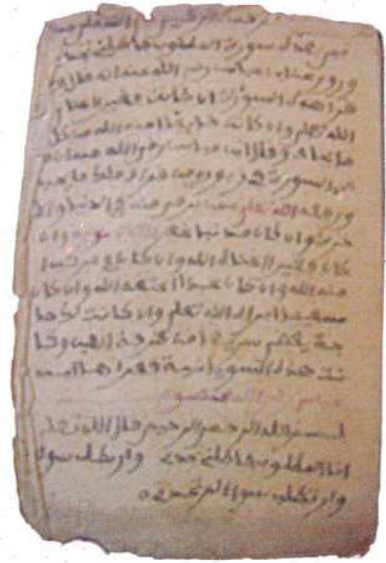

Source : Anonyme, Bibliothèque Mama Haidara, Tombouctou, Manuscrit $n^{\circ} 212,6$ pages

Fâtiha li jalb al arzâq, "Fâtiha pour "attirer les biens" »
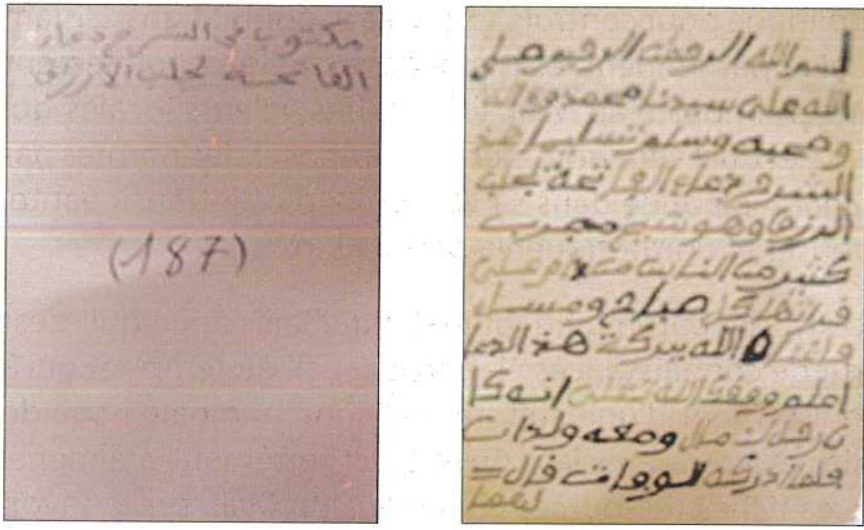

Source: Anonyme, Bibliothèque de Djenné, Manuscrit $n^{\circ} 187,21$ pages

Ces Fâtiha apparaissent finalement comme des ouvertures salvatrices à un moment de radicalisation et de fermeture de la pensée musulmane autour de préceptes étroits et intolérants. Elles sont la preuve de la capacité d'innovation et de ressourcement de l'islam, ici à travers la civilisation malienne. La mémoire plurielle de l'islam passe par la reconnaissance de cette diversité. 


\section{Conclusion}

Le Mali, terre d'accueil de l'islam depuis le VII' siècie, a su construire au fil des temps un savoir-vivre ensemble qui a toujours triomphé des conflits. La tolérance a toujours réussi à surpasser les différends et elle s'est imposée comme le maître mot de la vie en société. Ce vivre ensemble dans la tolérance réciproque a été mis à mal ces derniers temps par les conditions d'une globalisation débridée qui permet la circulation incontrôlée de toute sorte de produits dans une bande sahélienne devenue un espace d'opérations de puissances influentes, mais aussi de groupes plus ou moins téléguidés ou plus ou moins incontrôlés. Le Mali, et plus largement la zone sahélienne, sont devenus un espace privilégié du transit de drogue, d'armes et de trafics divers, dont celui d'êtres humains en déshérence, le tout sur fond de pauvreté endémique de la majorité des populations locales.

Les opérations de développement menées par "l'Occident » ont échoué, mais elles ont permis le déversement de fonds qui n'ont pas profité réellement à ceux auxçuels ils devaient aboutir. Des fonds arrivent désormais par le biais d'investisseurs qui utilisent la religion comme arme politique pour contrôler espaces et populations. L'islam politique auquel appartiennent les islamistes actuels est totalement extérieur, étranger, importé au Mali. II fait fi des valeurs locales qui gênent son assise et qu'il veut gommer pour imposer des normes par le biais d'une charia plaquée dans sa version la plus intransigeante, de façon à terroriser et domestiquer la population.

Dans ce contexte, le patrimoine pluriel du Mali, celui qui s'est construit à partir de connaissances élaborées au fil du temps et qui a permis l'épanouissement d'une culture malienne originale issue de traditions locales alliées à urı islam ouvert, est fortement malmené. S'appuyer sur ces riches ressources culturelles du Mali, sur le savoir être et le savoir vivre ensemble, sur la capacité à entretenir la cohésion sociale et donc à régler les conflits est un patrimoine commun qu'il importe de préserver. Tout cela doit permettre d'asseoir ce socle d'entente mutuelle entre les différents partenaires d'un Mali refondé autour d'un projet commun de développement issu de l'intérieur. 
Bibliographie

Al Karjousli, Soufian, 2006, La polysémie et le Coran, Lille, éd. Diffusion Anrt, Thèse à la carte, $351 \mathrm{p}$.

Al Karjousli, Soufian, 2009a, "De l'islam pluriel à l'islam "Mac Donald" », in A. Kiyindou, J.-C. Ekambo et L.-R. Miyouna (dir.), Communication et dynamiques de globalisation culturelle, Paris, L'Harmattan, pp. 73-86.

Al Karjousli, Soufian, 2009b, "La polysémie au cœur du dialogue interculturel et interconfessionnel, l'exemple de la traduction des vocables islâm et muslim dans le Coran ", Atelier de Traduction n`10, Actes du colloque international, Xème édition des Rencontres des traducteurs, "La traduction du langage religieux en tant que dialogue interculturel et interconfessionnel », Université de Suceava, 11-13 juillet 2008, Éditions Universitatii Suceava, Roumanie, pp. 77-91.

Al Karjousli, Soufian, 2010, «Les traversées de la Mer Rouge », in A. BlondelLoisel et R. Ranson (dir.), De l'art du passage, Histoire et représentations, Actes du colloque "Passages, Passerelles, Traversées », Faculté des Affaires Internationales, Université du Havre, 5-6 Novembre 2007, Paris, L'Harmattan, pp. 483-504.

Amselle, Jean-Loup, 1985, "Le wahabisme à Bamako (1945-1985) 》, Canadian Journal of African Studies, ${ }^{\circ} 19$ (2), pp. 345-357.

Bourdarias, Françoise, 2009, "Constructions religieuses du politique aux confins de Bamako (Mali) », Civilisations vol. 78, n², pp. 23-42. Mis en ligne le 30 décembre 2012, consulté le 24 juin 2014. URL : http://civilisations.revues. org/2070

Cissouma, Diama et Al Karjousli, Soufian, 2014, "Capter les richesses du patrimoine mondial dans une ville sainte de l'islam : la mise en tourisme a Djenné au Mali », Via@ - Revue internationale interdisciplinaire de tourisme $n^{\circ} 1$. Mis en ligne fin juin 2014, consulté le 24 juin 2014. URL : http://www. viatourismreview.net/

Cissouma, Diama et Ouallet, Anne, 2007, « Conservation du patrimoine et développement urbain à Djenné, Tombouctou et Gao au Mali », Mémoire de la ville, transmission des connaissances, des savoirs et des cultures, Paris, CIDEF/AFI, pp. 69-84.

Cissouma, Diama et Ouallet, Anne, 2009, « Le patrimoine comme vecteur de la globalisation et du développement local : I'exemple d'espaces maliens et éthiopiens patrimonialisés », in A. Kiyindou, J.-C. Ekambo et L.-R. Miyouna (dir.), Communication et dynamiques de globalisation culturelle, Paris, L'Harmattan, pp. 49-64. 
Di Méo, Guy, 1998, Géographie sociale et territoires, Paris, Nathan.

Haidara, Ismaël Diadié, 1999, Les Juifs à Tombouctou, recueil des sources écrites relatives au commerce juif à Tombouctou au $19^{\text {ème }}$ siècle, éd. Donniya.

Hentati, Nejemedine, 2004, Le malékisme dans l'Occident musulman jusqu'au VIe/XIe siècle [Al mazhab al mâliki bi-I gharb al islâmi], Tunis, L'Or du Temps [Tabr ez Zaman].

Holder, Gilles, 2004, "La cité comme statut politique : places publiques, pratiques d'assemblées et citoyenneté au Mali », Journal des Africanistes, Cité-État et statut politique de la ville en Afrique et ailleurs, Tome 74, fasc. 1-2, pp. 56-96. Mis en ligne le 15 mars 2007, consulté le 24 juin 2014. URL : http:// africanistes.revues.org/537

Holder, Gilles (dir.), 2009, L'islam, nouvel espace public en Afrique, Paris, Karthala.

Hunwick, John (ed.), 1985, Shari'a in Songhay: the replies of al-Maghili to the questions of Askia Al-hajj Muhammad, Londres, Oxford University Press.

Ibn Fares, [mort en 1004], Kitâb as sâhibi fy fiqh al lugha al arabyia wa masâiliha wa sunan al arab, ("Le live du compagnon sur le savoir linguistique de la langue arabe et de ses règles langagières »), retravaillé par Muhammad Ali Baydun, 1997, éd Muhamed Ali Baydun, dar el kutub el 'ilmyia , Liban.

Robinson, David et Triaud, Jean-Louis (dir.), 1997, Le temps des marabouts, itinéraires et stratégies islamiques en AOF, v. 1880-1960, Paris, Karthala.

Ziyadé, Nicolas, 1991, Ifryqyiât Dirâsât fy-l Maghreb al 'Arabî was-Sûdân al gharbî, [" Des écrits sur l'Afrique : études sur le Maghreb arabe et de l'ouest du Soudan »], Londres, Riyad er-Rayes. 
li contemporain ? II est bien différent des images que les médias diffusent ! iété malienne a considérablement évolué depuis l'indépendance, les jeunes beaucoup plus nombreux et mieux informés, les femmes plus actives. Mais angements qui ont marqué notamment la gouvernance et la situation mique paraissent décalés au regard des nouvelles contraintes, attentes et ces liées à ces mutations. Comment cette société, dans sa diversité, t-elle et s'engage-t-elle dans la modernité ? Voilà le sujet de ce livre.

inquantaine de chercheurs en sciences sociales, dans le cadre d'une sration franco-malienne, ont travaillé sur le Mali contemporain dans la e qui a immédiatement précédé la crise (2007-2012). Ils se sont Ialement intéressés à la vie politique dans les communes et quartiers, à la de la décentralisation, à l'image que l'Etat en action donne de lui, au fait ux dans ses manifestations et ses tendances lourdes, mais aussi aux divers 5 de la migration, et enfin et surtout à la place des jeunes dans la société.

eau qui se dégage de leurs observations est sans complaisance : faire évoluer ement la situation actuelle de la société malienne est un défi pour les décideurs emble de la population ; et pour y parvenir, il est impérieux d'ébranler les zurs, blocages, et faux-fuyants qui ont conduit le pays au bord du gouffre.

\section{teurs}

In AL KARJOUSLI

(Issaka) BAGAYOGO ma Fasséry BALLO que BERTRAND tienne BIDOU 3LANKESPOOR ר BRUNET-JAILLY es CHARMES na CISSE

CISSOUMA TOGOLA 'aul COLLEYN

OULIBALY COULIBALY n COULIBALY sphe DAUM
- Mahamadou DIAWARA

- Elina DJEBBARI

- Anne DOQUET

- Isabelle DROY

- Assa GAKOU DOUMBIA

- Rémi GENEROSO

- Vincent GERONIMI

- Gilles HOLDER

- Youssouf KAREMBE

- Yada KASSOGUE

- Amadou KEITA

- Amaga Doguélou KODIO

- Doulaye KONATE

- Stéphanie LIMA

- Claire MAINGUY
- Sandrine MESPLÉ-SOMPS

- Pierre MORAND

- Emmanuelle OLIVIER

- Anne OUALLET

- Pierre PRUD'HOMME

- Anne-Sophie ROBILLIARD

- Harris SELOD

- Famory SINABA

- Sadio SOUKOUNA

- Mamy SOUMARE

- Moussa SOW

- Arouna SOUGANE

- Gilles SPIELVOGEL

- Céline THIRIOT

- KadariTRAORÉ 